\title{
Energy-Based Plasmonicity Index to Characterize Optical Resonances in Nanostructures
}

\author{
Marvin M. Müller,* Miriam Kosik,* Marta Pelc, Garnett W. Bryant, Andrés Ayuela, Carsten Rockstuhl, \\ and Karolina Słowik
}

\begin{abstract}
Resonances sustained by plasmonic nanoparticles provide extreme electric field confinement and enhancement into the deep subwavelength domain for a plethora of applications. Recent progress in nanofabrication made it even possible to tailor the properties of nanoparticles consisting of only a few hundred atoms. These nano particles support both single particle like resonances and collective plasmonic charge density oscillations. Prototypical systems sustaining both features are graphene nanoantennas. In pushing the frontier of nanoscience, traditional identification, and classification of such resonances is at stake again. We show that in such nanostructures, the concerted electron cloud oscillation in real space does not necessarily come along with collective dynamics of conduction band electrons in energy space. This unveils an urgent need for a discussion of how a

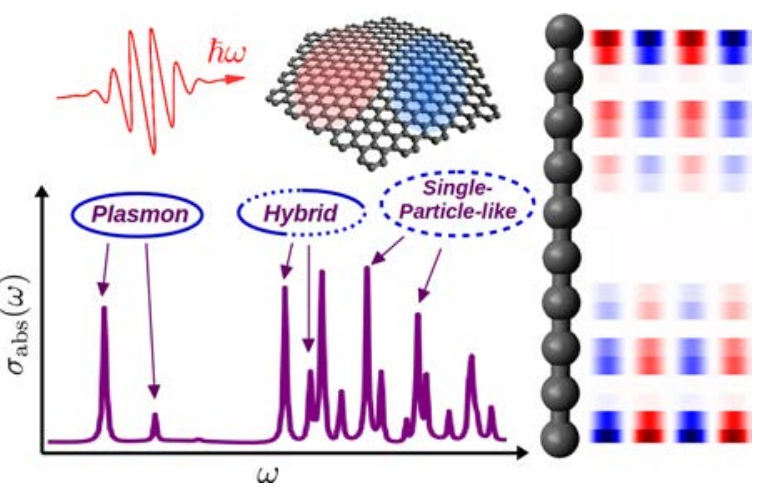
plasmon in nanostructures should be defined. Here, we propose to define it relying on energy space dynamics. The unambiguous identification of the plasmonic nature of a resonance is crucial to find out whether desirable plasmon assisted features, such as frequency conversion processes, can be expected from a resonance. We elaborate an energy based figure of merit that classifies the nature of resonances in nanostructures, motivated by tight binding simulations with a toy model consisting of a linear chain of atoms. We apply afterward the proposed figure of merit to a doped hexagonal graphene nanoantenna, which is known to support plasmons in the near infrared and single particle like transitions in the visible.
\end{abstract}

\section{INTRODUCTION}

Metallic nanoparticles of various sizes and shapes have been the workhorse for plasmonics in the last decades. ${ }^{1-7}$ There, plasmons, the quanta of coherent electronic charge density oscillations, are coupled to light, giving rise to hybrid excitations of photons strongly interacting with conduction electrons. Plasmonic resonances have the extraordinary ability to confine, focus, and enhance electromagnetic energy into subwavelength spatial volumes near the edges and tips of particles far below the diffraction limit. ${ }^{6,8-10}$ Nonlinear optical effects, like higher harmonic generation ${ }^{11-13}$ with applications in plasmonic sensing, ${ }^{14-16}$ for instance, are expected to be promoted by plasmonic resonances as well. Further applica tions of plasmonic devices can be found in the design of optical metamaterials ${ }^{17,18}$ and single photon sources, ${ }^{19,20}$ photo detection, ${ }^{21-25}$ and photothermal medicine, such as cancer therapy, ${ }^{26-28}$ and many more interdisciplinary fields of research.

Having novel top down and bottom up fabrication processes at hand, ${ }^{29}$ the field of plasmonics recently experienced a boost toward the true nanoscale. ${ }^{5,10,30-34}$ In nanoplasmonics, single quantum emitters, such as quantum dots, ${ }^{35,36}$ small mole cules, ${ }^{37}$ or optically active impurities in solids, ${ }^{38}$ which are placed in the vicinity of the nanoparticle's hotspots, can be addressed individually. In this tailored photonic environment in proximity to the nanoparticle, the emitter's properties like its spontaneous emission rate, for instance, can be significantly enhanced. ${ }^{39}$ However, in shrinking the size of plasmonic devices into the nanoscale region, one has to take into account the finite size of the particles. In the context of graphene nanoplasmonics, it has been shown that for antennas with characteristic sizes below $10 \mathrm{~nm}$ a homogenous description of the material at the local level, using, for example, a Drude model, fails to describe the plasmonic behavior properly. ${ }^{40,41}$ For such small structures, finite size quantization effects become important and are not taken into account by simple continuum models. ${ }^{42}$ Although classical approaches can be 
surprisingly accurate in describing localized surface plasmon resonances even at the angstrom scale, ${ }^{43-45}$ it is inevitable that atomistic quantum mechanical models are needed to capture energy space dynamics as well. Methods of choice are the jellium model, ${ }^{46}$ quantum fluid dynamics, ${ }^{47-49}$ the config uration interaction method, ${ }^{50,51}$ the Bethe-Salpeter ap proach, $^{52-54}$ density functional theory (DFT), ${ }^{55-71}$ or tight binding (TB) based approaches. ${ }^{72-77}$ In this work, we employ the latter to find the electronic energy landscape of the systems under consideration. The transition from homogeneous solid state like nanoantennas to molecular clusters of only dozens or hundreds of atoms can be related to the electronic structure of the system. When the solid state system gets smaller, the valence and conduction bands undergo a discretization process into energy levels. Consequently, terms like valence band, conduction band, band gap, and Fermi energy are not well defined any more in a strict sense. In a discrete energy landscape, one should instead rather speak of the highest occupied molecular orbital (HOMO) and lowest unoccupied molecular orbital (LUMO) to specify the occupation characteristics of the electronic energy states in the system. The terminologies of physical chemistry, solid state physics, and quantum optics merge in nanoplasmonics. To keep language simple and for illustration purposes, we speak in this work of Fermi energy even in systems with discrete energy states. If the HOMO state is not fully occupied, we identify the Fermi energy with the HOMO energy level. In the case of fully occupied HOMO, we locate the Fermi energy around the middle of HOMO and LUMO. However, we emphasize that the Fermi energy itself never enters any simulation as an input parameter but only the discrete number of doping electrons does.

Not only the electronic energy levels but also the electron dynamics and, consequently, the optical excitation processes of such nanoscaled systems are affected by their size. The transition that a metallic continuous band structure undergoes toward a discretized molecular Jabłonski diagram is reflected in the nature of the resonances found in the absorption spectrum as well, when the system is miniaturized. In this work, we observe a coexistence of both single particle like resonances known from small molecular atomic clusters and plasmonic resonances found in metals that depend on the interaction energy among the collection of conduction electrons contributing to the resonance. The establishment of a figure of merit that allows one to conclude whether a resonance is single particle like or plasmonic in nature is at the heart of this contribution. This prompts simultaneously for further discussion of specific characteristics of such excitations at the nanoscale.

The former class of single particle like resonances can be directly related to a transition in the energy landscape from an occupied energy state to an unoccupied one, as shown in Figure 1a. They occur at distinct excitation frequencies and do not depend on the number of doping electrons in the system in the TB formalism employed here. In this approach, the energy landscape and the single particle like resonances are not affected by doping directly, unless the doping electron in question populates an energy state that was the acceptor state for the transition before. In this case, the resonance vanishes from the spectrum because the transition got blocked. This effect is known as Pauli blocking. ${ }^{78}$ We want to emphasize that the independence of the energy landscape on the number of (a) Single-Particle

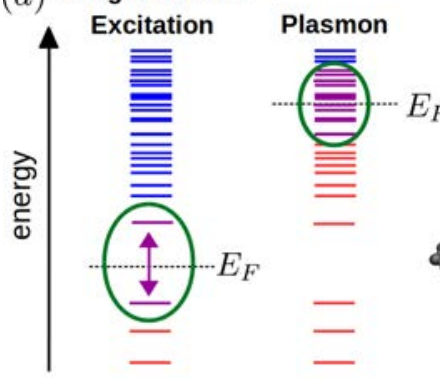

(b)

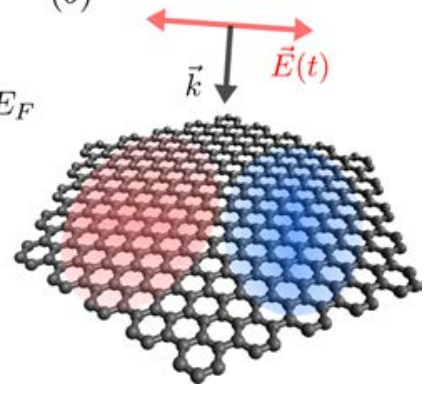

Figure 1. (a) Schematic illustration of the energy space dynamics of single particle like and plasmonic transitions. Whereas the energy of a single particle like resonance is determined by the energy landscape of the nanosystem, the plasmonic resonance energy is determined by the interaction energy of many electrons just above and below the Fermi energy and cannot be explained by the energy landscape of the system only. (b) Sketch of the hexagonal GNA under consideration.

doping electrons is, especially for small systems, not a valid assumption in the strict sense but merely a result of the model.

The latter class of resonances, known as plasmons in metals, emerges in a complex interplay of interacting electrons which dynamically populate and depopulate energy states around the Fermi energy. This oscillatory behavior was previously found in time dependent DFT (TDDFT) simulations of small metallic spheres by Townsend and Bryant and was named "sloshing". ${ }^{59,60}$ Resonances of this nature are determined by the single electronic transitions near the Fermi energy strongly dressed by the interaction of a collection of electrons that populate states near the Fermi energy. As a result, one cannot predict the spectral position of these collective resonances by inspecting energy differences of electronic states in the energy landscape of the system. The spectral position of these resonances is also influenced by the interaction energy of the electrons in the system. Therefore, such resonances are sensitive to the number of doping electrons because the position of the Fermi energy is modified and different states contribute to the resonance. This shift of the absorption peak for collective resonances under varying doping conditions has been predicted in doped graphene nanoantennas (GNAs) by Thongrattanasiri, Manjavacas et al. before. ${ }^{40,41}$ Furthermore, Cox and Garcia de Abajo have conducted comprehensive studies to characterize the optical response of these structures. ${ }^{13,77,79-81}$ Because of their promising applications and the high degree of control over their properties, GNAs are an optimal showpiece to demonstrate the applicability of our figure of merit.

Polycyclic aromatic hydrocarbon molecules ${ }^{82,83}$ and GNAs ${ }^{72,73,75,84}$ constitute versatile elements in the nano photonics toolbox and exhibit the above outlined hybrid character of both plasmonic and single particle like resonances. Their electronic structures have been studied very intensely in the last decades. They have fascinating properties that unlock a plethora of linear and nonlinear applications both in the classical and the quantum world. Unique to nanoantennas made from graphene, which distinguishes them from their noble metal counterparts, is that their properties can be modified significantly a posteriori to the fabrication process by electrostatic gating. ${ }^{73}$ This renders them promising candidates for quantum information processing, ${ }^{85}$ quantum sensing applications, ${ }^{86}$ and nonlinear quantum optics in general. ${ }^{81}$ Even a dynamic tuning of their properties in time comes into 
reach thanks to ultrafast optical pumping and probing techniques that modify the carrier density distribution on the timescale of femtoseconds. ${ }^{87-91}$

We notice the coexistence of both classes of resonances in the absorption cross section of the same nanostructure simultaneously, as shown in Figure 1. To take advantage of the above outlined beneficial plasmonic effects, it is desirable to classify these resonances and to have a tool at hand that distinguishes between single particle like and plasmonic response. Therefore, we present an energy based plasmonicity index (EPI) that is a normalized figure of merit for the nature of resonances in molecular structures, which is independent of the relative strength of the resonance in the spectrum.

Our paper is organized as follows. We first introduce the tight binding based electronic structure model and the time propagation simulation method. To construct a figure of merit that may distinguish the single particle like and plasmonic nature of resonances, we demonstrate the real space and energy space fingerprints of both classes of resonances on simple toy models. From a mere inspection of the energy landscapes of these toy models, we can directly deduce the nature of a resonance in the absorption spectrum and can use it to demonstrate the applicability of the EPI. To this end, we adduce the (finite) linear atomic chain and the (finite) dimerized linear atomic chain as prototypes for metallic and semiconducting systems, respectively. Their consideration serves to sharpen the EPI before we utilize it to analyze the spectrum of a hexagonal GNA.

\section{METHODS}

In the following, we briefly introduce our methodology. Along the lines of a previous work from Thongrattanasiri, Manjavacas, Cox, and Garcia de Abajo, ${ }^{40,41,77}$ the nano antennas are described in the TB framework. The coupling to an externally applied laser pulse is done in the quasistatic limit and dipolar approximation. From the TB energy states, we construct the ground state density matrix of the antenna and propagate it through time, applying a master equation under the influence of the illumination laser. Dissipation is taken into account by including a phenomenological damping term forcing the system back into its ground state. The details of this description are documented in the following sections.

Nanoantennas. The nanoantennas under consideration are modeled relying on the TB approach. While this is a generic model applicable to many systems, we bias the choice of parameters toward our system of interest, that is, GNAs. It is assumed that two of the six electrons per carbon atom are bound tightly by the carbon atom in its 1 s orbital. Three electrons per carbon atom in hybridized $2 \mathrm{sp}^{2}$ orbitals form strong in plane $\sigma$ bonds. They are shared among neighboring carbon atoms. The remaining electron populates an out of plane $\mathrm{p}_{z}$ orbital, which is localized at the respective position of the atom it belongs to, and which can form a $\pi$ bond with the $\mathrm{p}_{z}$ orbital of a neighboring atom. Electrons in $\pi$ bonds are highly mobile, thus capable of hopping to neighboring carbon atom sites and participating in conduction. These weak $\pi$ bonds serve as a channel for electronic transport in the nanoantenna. It is quantified by a hopping parameter $t$, that allows electrons to hop from one atomic site 1 to another neighboring site $\mathrm{l}^{\prime}$ and back again. The Hamiltonian of such a system reads

$$
H_{\mathrm{TB}}=-t \sum_{\left\langle l, l^{\prime}\right\rangle}\left(|l\rangle\left\langle l^{\prime}\right|+\text { h. c. }\right)
$$

where "h.c." stands for the Hermitian conjugate. Throughout this work, we use $t=2.66 \mathrm{eV}^{92}$ as the hopping parameter between neighboring atomic $\mathrm{p}_{z}$ orbitals in graphene and $\left\langle l, l^{\prime}\right\rangle$ means summation over the nearest neighbor atomic sites $l$ and $l^{\prime}$ only. The geometry of the flake thus defines $H_{\text {Тв }}$ entirely and upon diagonalization, we obtain the set of $N$ energy eigenstates $\{|j\rangle\}$ and eigenenergies $\left\{E_{j}\right\}$ with $H_{\mathrm{TB}}|j\rangle=E_{j}|j\rangle$, where $j \in[0, N$ - 1] on a flake with $N$ atoms. Expansion of these energy eigenstates into the basis set of localized atomic site $\mathrm{p}_{z}$ orbitals $\{|l\rangle\}$ according to $\left.|j\rangle=\sum_{1} a_{j} l l\right\rangle$ allows to visualize the single particle energy eigenstates in real space on the flake. According to the aufbau principle of quantum chemistry, we define the ground state density matrix $\rho^{0}$ of the antenna with $N_{\mathrm{e}} \mathrm{p}_{z}$ electrons as

$$
\rho^{0}=\frac{2}{N_{\mathrm{e}}} \sum_{j} f_{j}\left(N_{\mathrm{e}}\right)|j\rangle\langle j|
$$

where $f_{j}\left(N_{\mathrm{e}}\right) \in[0,1]$ is the Fermi-Dirac distribution that determines how many electrons per spin occupy the state $|j\rangle$. For an undoped antenna, this corresponds to an equal distribution of the electrons among all the atoms in the antenna. For more details on the TB method applied to graphene, we direct the interested reader to further literature. ${ }^{93}$ The way this initial density matrix configuration evolves dynamically under laser illumination is outlined in the following section.

Coupling to Laser Illumination. To investigate the antenna's response to external laser illumination, we apply a dipolar coupling scheme in the quasistatic limit, where the electric field of the laser pulse is coupled to the nanoantenna according to

$$
H(t)=H_{\mathrm{TB}}-e \varphi(t)=H_{\mathrm{TB}}-e\left[\varphi^{\mathrm{ext}}(t)+\varphi^{\text {ind }}(t)\right]
$$

Here, $\varphi_{l}^{\text {ext }}(t)=-\mathbf{r}_{l} \cdot \mathbf{E}(t)$ is the externally applied electric potential at atomic site $l$ at position $\mathbf{r}_{l}$ that arises through the external electric laser field $\mathbf{E}(t)$. Throughout the whole paper, we work in the linear response regime. The induced potential at site $l$ is determined by taking into account the Coulomb interaction between electrons located at different sites $l$ and $l^{\prime}$

$$
\varphi_{l}^{\text {ind }}(t)=-e N_{\mathrm{e}} \sum_{l^{\prime}} v_{l l^{\prime}}\left(\rho_{l^{\prime} l^{\prime}}(t)-\frac{1}{N}\right)
$$

Here, $v_{l l^{\prime}}$ is the Coulomb interaction matrix element that couples electrons at atomic sites $l$ and $l^{\prime}$, and $-e N_{\mathrm{e}}\left(\rho_{l l}(t)-1 /\right.$ $N)$ is the induced charge accumulated on the atomic site $l$. For atomic sites far away from each other, we employ the usual $1 / r$ scaling law for the values in $v$. For the onsite, nearest neighbor, and next to nearest neighbor values, we use Coulomb interaction values calculated by Potasz et al. ${ }^{84}$ both in the GNA and the atomic chains.

Time Propagation. To propagate the nanoantenna's state $\rho(t)$ through time, we employ the master equation

$$
\frac{\partial}{\partial t} \rho(t)=-\frac{i}{\hbar}[H(t), \rho(t)]-\frac{1}{2 \tau}\left(\rho(t)-\rho^{0}\right)
$$

The second term on the right hand side is a phenomeno logically introduced damping term that causes an exponential decay of the system toward its ground state $\rho^{0}$ with a scattering time $\tau \approx 100 \mathrm{fs}$, motivated by the scattering time of electrons 
in extended 2D graphene sheets. ${ }^{94}$ This scattering time reflects both radiative and nonradiative losses because of optical phonons, for instance. The quantities that are analyzed in time are the populations of the density matrix in both energy basis $\{|j\rangle\}$ and atomic site basis $\{|l\rangle\}, \rho_{j j}(t)$ and $\rho_{l l}(t)$, respectively. Furthermore, we record the induced dipole moment $\mathbf{p}(t)=-$ $e N_{\mathrm{e}} \sum_{l} \mathbf{r}_{l}\left(\rho_{l l}(t)-\rho_{l l}^{0}\right)$ to calculate the absorption cross section of the antenna. In particular, we excite the system with a short, spectrally broad, linearly polarized electric pulse and obtain the linear polarizability $\alpha_{x}(\omega)=p_{x}(\omega) / E_{x}(\omega)$ (for $x$ polarized light) as a function of frequency $\omega$, which allows for determining the absorption cross section $\sigma_{x, \mathrm{abs}}(\omega)=\omega$ $\operatorname{Im}\left[\alpha_{x}(\omega)\right] / \varepsilon_{0} c_{0}{ }^{10}{ }^{10}$ where $c_{0}$ is the speed of light in free space and $\varepsilon_{0}$ is the vacuum permittivity.

\section{RESULTS AND DISCUSSION}

Prior to applying the methodology to GNAs, we explore two toy models with well known electronic structure: the linear atomic chain and the dimerized linear atomic chain. These toy models constitute prototypes for metallic and semiconducting behavior, respectively. ${ }^{60,62,68,69,95,96}$ The band structure of the former consists of densely lying electronic energy states, effectively acting as a metallic conduction band which is half occupied. In the band structure of the dimer chain, an energy gap opens up around the Fermi energy at $E_{\mathrm{F}}=0$, effectively separating densely lying states below it (valence band) from densely lying states above it (conduction band). This band structure is typical for semiconductors. Once, we have identified the basic signatures for the respective type of resonance, we can derive our figure of merit that allows to distinguish these resonances, so we can apply it afterward to the GNA.

Linear Chain. The linear atomic chain constitutes a one dimensional prototype of a metallic system supporting plasmonic resonances. In this study, $N=70$ atoms are coupled to their nearest neighbors by a hopping parameter $t=2.66 \mathrm{eV}$. Its energy landscape is shown in Figure 2a. It exhibits equally spaced densely lying electronic states around the Fermi energy without a band gap. The set of states originates from the discretization of a single metallic conduction band, halfway
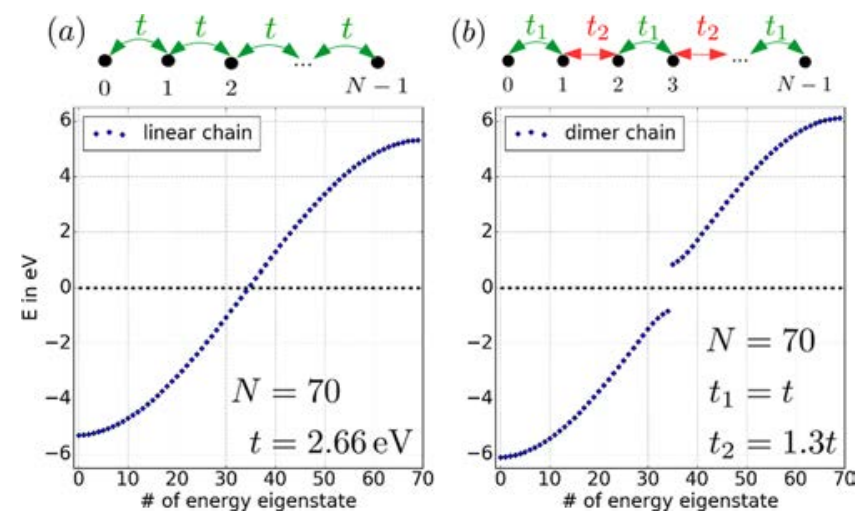

Figure 2. Energy landscapes of the linear and the dimer chain: (a) $N$ $=70$ atoms of the linear chain are coupled with a hopping parameter $t$ to their nearest neighbors. Below, the energy landscape of the energy eigenstates is shown. The dashed horizontal line denotes the Fermi energy in the case of no doping electrons on the chain. (b) In the dimerized chain, the $N=70$ atoms are coupled to their neighbors by two different hopping parameters $t_{1}$ and $t_{2}$. As a consequence, a band gap opens around $E=0$ and renders the system semiconducting. filled by electrons in the neutral antenna. In the undoped system $(d=0)$, the Fermi energy lies at $E_{\mathrm{F}}=0$, the HOMO energy is $E_{\mathrm{H}}=-0.12 \mathrm{eV}$, and, for symmetry reasons, the LUMO has the energy $E_{\mathrm{L}}=\left|E_{\mathrm{H}}\right|$. By doping the system with additional electrons, the Fermi energy increases because more and more states above $E=0$ are populated. Figure 3a shows
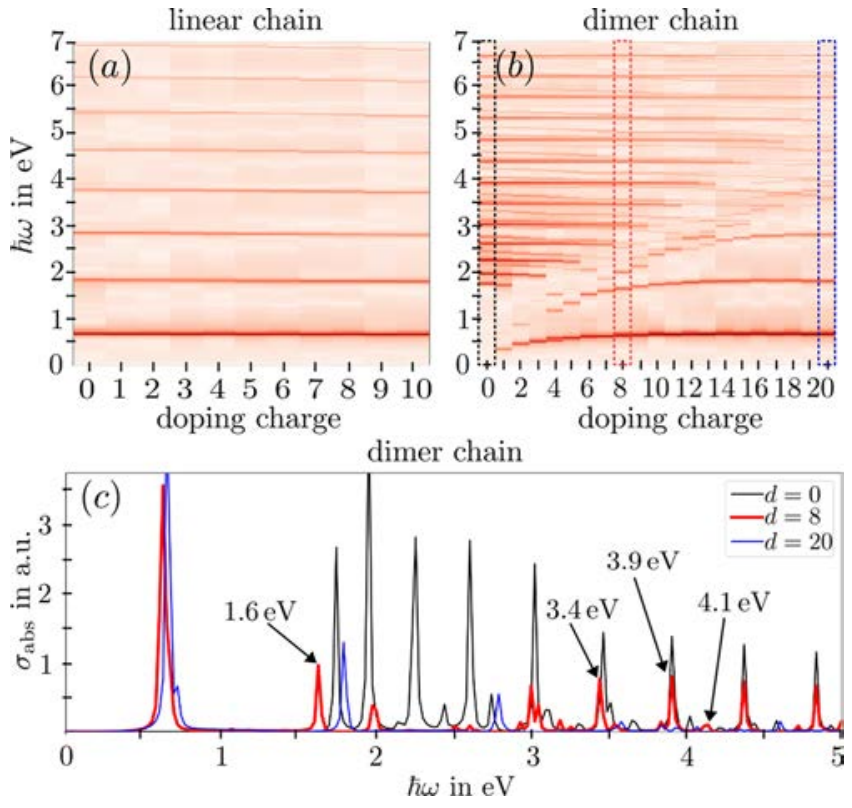

Figure 3. Absorption cross sections of the 70 atomic linear chain (a) and dimer chain (b) for various doping levels. (c) Absorption cross section of the dimer chain for 0,8 , and 20 doping electrons. The arrows indicate the resonances that are investigated in more detail.

the linear absorption cross section of the linear chain for various doping levels. It reveals that the optical properties of the linear chain do not change with doping in the investigated range.

This is understandable because the local electronic energy landscape around a Fermi energy of, for example, $E_{\mathrm{F}}=1 \mathrm{eV}$ (corresponding to 10 doping electrons) does not differ from the one around $E_{\mathrm{F}}=0$ in the undoped case. In both cases, the Fermi energy lies inside the conduction band, a region of densely lying energy states. Important for the electron dynamics and, consequently, for the optical properties is the fact that above (below) the Fermi energy there are plenty of unoccupied (occupied) states. Therefore, we expect plasmonic resonances in the linear chain independent of the doping level.

To investigate the fingerprints of plasmonic resonances in energy space, we study the density matrix elements of the linear chain. In this example, the linear chain has been doped with 8 electrons. Identical results can be found for other moderate doping levels. Figure 4 shows the absolute value of the linear chain's stationary state density matrix elements in the energy basis near the Fermi energy after being subject to continuous wave (CW) illumination for excitation frequencies of $\hbar \omega=1.8 \mathrm{eV}$ (a) and $\hbar \omega=6.2 \mathrm{eV}$ (b), respectively. These frequencies coincide with resonances of the linear chain (see Figure 3a). Please note, throughout this work we always deplete the diagonal of the density matrices under consid eration. The figure of merit to be developed below depends only on the off diagonal elements, as shown in the figures. We observe that several transitions near the Fermi energy are involved in the resonance. The interaction energy of the 


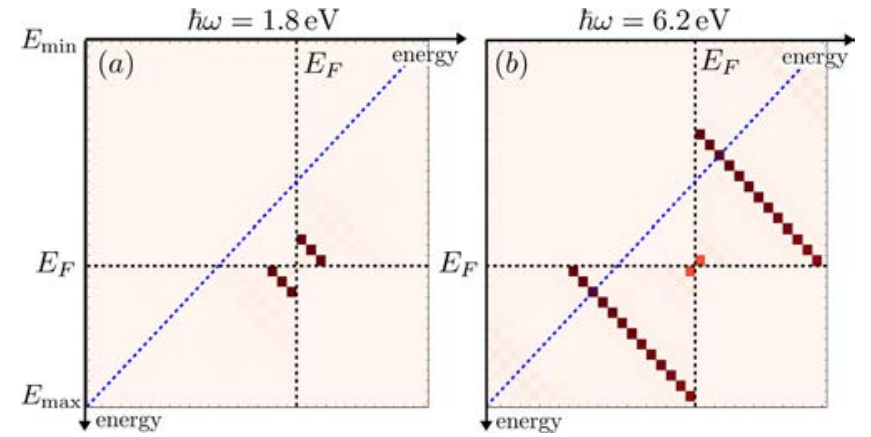

Figure 4. Absolute value of the density matrix elements of a linear chain doped with 8 electrons under $\mathrm{CW}$ illumination after about 30 optical cycles. The illumination frequency is $\hbar \omega=1.8 \mathrm{eV}$ (a) and $\hbar \omega$ $=6.2 \mathrm{eV}(\mathrm{b})$, respectively.

electrons determines the spectral position of the resonance, revealing its collective nature. Moreover, it is evident that the Fermi energy's position determines which transitions contrib ute to the resonance. For the undoped linear chain, all the results mentioned above are qualitatively the same. The only difference is that the Fermi energy will be at $E=0$, such that the two black dashed horizontal and vertical lines (signifying $\left.E_{\mathrm{F}}\right)$ and the blue dashed diagonal line, as shown in Figure 4, intersect at a single point. The states involved in the resonances will be symmetric with respect to the anti diagonal blue dashed line of the density matrix, which is not the case in doped systems. In real space, the prominent resonances in the spectrum, as shown in Figure 3a, correspond to the first (third, fifth, seventh, ...) plasmonic mode exhibiting $1(3,5,7, \ldots)$ node $(\mathrm{s})$ in the induced charge oscillation pattern (not shown in the figures).

Dimer Chain. The dimer chain is a one dimensional arrangement of atoms being coupled to their nearest neighbors asymmetrically. In this study, the couplings are such that evenly labeled atoms are coupled to their right neighbors by a coupling constant $t_{1}=t$ and to their left neighbors by a coupling constant of $t_{2}=1.3 t$. The results shown in this work depend only quantitatively on the choice of $t_{2}>t_{1}=t$. The energy landscape is shown in Figure $2 \mathrm{~b}$. It exhibits a similar overall shape as compared to the linear chain. However, there is one clearly distinguishing feature: a band gap opens up symmetrically around $E=0$. The set of states below (above) the band gap originates from the discretization of the valence (conduction) band, completely populated (unpopulated) by electrons in the undoped case. Consequently, the undoped system should not show any metal like collective plasmonic resonances. We expect mostly single particle like behavior for excitation frequencies that can lift electrons across the band gap $E_{\mathrm{BG}} \approx 1.67 \mathrm{eV}$, that is, for $\hbar \omega>E_{\mathrm{BG}}$ only. This interpretation of the electronic landscape is in good agreement with the absorption cross section of the structure, as shown in Figure $3 \mathrm{~b}, \mathrm{c}$. For $d=0$, there are no resonances below $E=1.67$ $\mathrm{eV}$, which represents the dipole active HOMO-LUMO transition resonance. For two additional doping electrons, this HOMO-LUMO transition peak disappears in the absorption spectrum because the former LUMO state is now fully occupied and cannot serve as an acceptor state any more. This behavior of vanishing resonance peaks repeats consec utively when adding electrons in pairs and filling up more states. We can see the abovementioned Pauli blocking mechanism here, revealing the single particle like nature of the resonance.

Figure 5 shows the absolute values of the density matrix elements in the energy basis of the dimer chain in the undoped
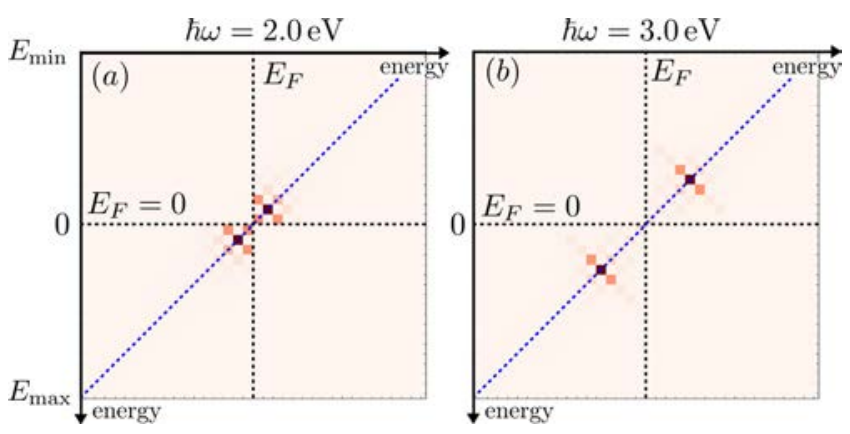

Figure 5. Absolute value of the density matrix elements in the energy basis of the dimer chain in the undoped case under CW illumination in the stationary state. The illumination frequency is (a) $\hbar \omega=2.0 \mathrm{eV}$ and (b) $\hbar \omega=3.0 \mathrm{eV}$, respectively.

case under $\mathrm{CW}$ illumination for the two selected resonance frequencies (all the other resonances behave qualitatively the same). In contrast to the previously considered linear chain, only transitions from state $\mathrm{m}$ to state $\mathrm{n}$, which meet the energy matching condition $\left|E_{\mathrm{m}}-E_{\mathrm{n}}\right| \approx \hbar \omega$, participate in the electron dynamics, revealing the single particle like behavior of the resonance.

As soon as we dope the dimer chain, additional resonances in the low energy region appear (see Figure $3 b$ ). They blue shift with increasing doping until they saturate in close vicinity to those spectral positions, where the linear chain's resonances are located (see Figure 3a,b). For the latter reason, we deem them of plasmonic nature. This interpretation is supported by inspecting the energy landscape of the dimer chain. Doping the structure with additional electrons changes the local electronic landscape environment around the Fermi energy dramatically. The latter is lifted from inside the band gap into the conduction band, such that the dimer chain becomes metallic and conduction band electrons can move collectively and consequently form plasmons.

The investigation of the density matrix elements after CW illumination on resonance is shown in Figure 6 for a predominantly collective (a), two predominantly single particle like (b,c), and a hybrid resonance (d). The collective resonance at $\hbar \omega=1.6 \mathrm{eV}$ and the hybrid resonance at $\hbar \omega=$ $4.1 \mathrm{eV}$ couple states from just below the Fermi energy to states just above it, whereas in the single particle like resonances at $\hbar \omega=3.4 \mathrm{eV}$ and $\hbar \omega=3.9 \mathrm{eV}$, only the states that meet the energy matching condition $\left|E_{\mathrm{m}}-E_{\mathrm{n}}\right| \approx \hbar \omega$ are coupled, disregarding the position of the Fermi energy in the system. Next, we would like to explore signatures of these resonances in real space and argue that from the real space dynamics the character of the resonances cannot always be clearly seen.

Real Space Dynamics. In real space, one can find the fingerprints of collective, single particle like, and hybrid resonances in the pattern of the induced charges on the atoms of the chain. Figure 7 shows the induced charge on the dimer chain's atomic sites as a function of time under CW illumination on resonance after about 30 optical cycles for the four frequencies already investigated, as shown in Figure 6. We show the last five optical cycles of the simulation period. It is apparent that the predominantly collective resonance at $\hbar \omega=$ 

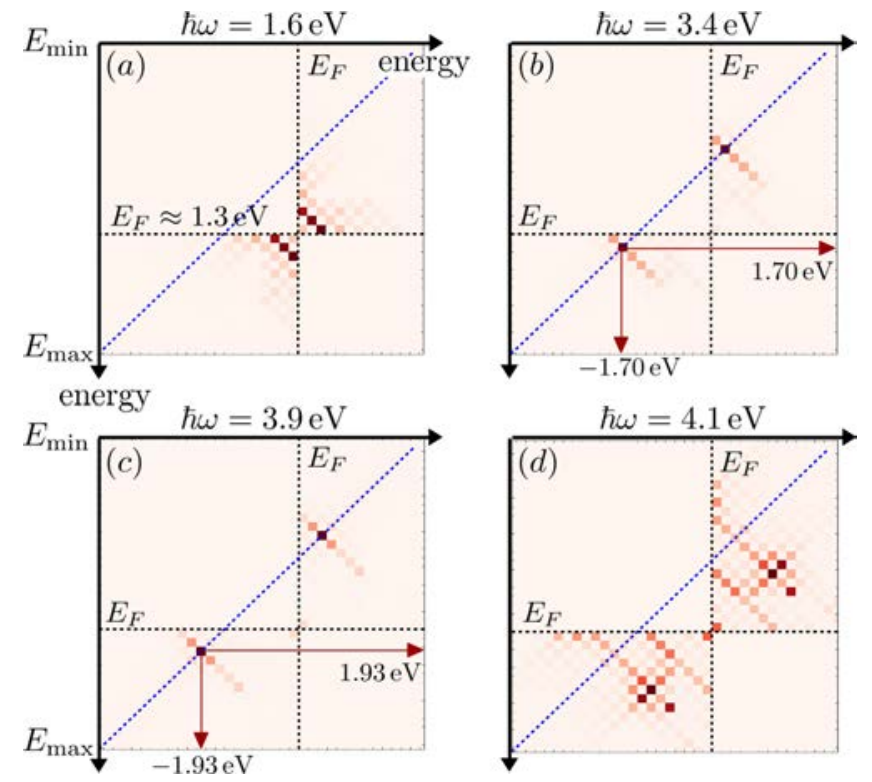

Figure 6. Absolute value of the density matrix elements in the energy basis of the dimer chain doped with 8 additional electrons under CW illumination in the stationary state. The illumination frequencies are chosen such that one of the resonances shows collective behavior, $\hbar \omega$ $=1.6 \mathrm{eV}$ in (a), two show single particle like behavior, $\hbar \omega=3.4 \mathrm{eV}$ in (b) and $\hbar \omega=3.9 \mathrm{eV}$ in (c), and one is of hybrid character, $\hbar \omega=4.1$ $\mathrm{eV}$ in $(\mathrm{d})$.

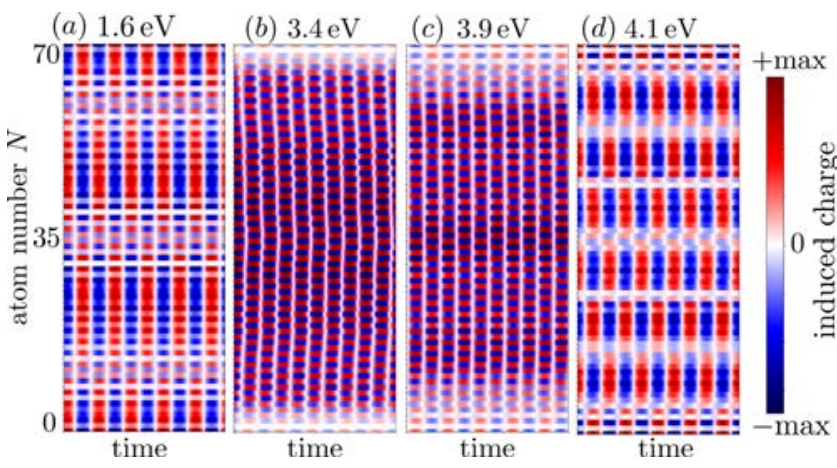

Figure 7. Induced charge on the dimer chain doped with 8 additional electrons under $\mathrm{CW}$ illumination in the stationary state for the last five optical cycles of the simulation period. The illumination frequencies coincide with those in Figure 6 and are chosen such that one of the resonances shows collective behavior, $\hbar \omega=1.6 \mathrm{eV}$ in (a), two show single particle like behavior, $\hbar \omega=3.4 \mathrm{eV}$ in (b) and $\hbar \omega=3.9 \mathrm{eV}$ in (c), and one is of hybrid character, $\hbar \omega=4.1 \mathrm{eV}$ in (d).

$1.6 \mathrm{eV}$ and the hybrid one at $\hbar \omega=4.1 \mathrm{eV}$ with their long range standing wave oscillation patterns can be easily told apart from the single particle like resonances at $\hbar \omega=3.4 \mathrm{eV}$ and $\hbar \omega=3.9$ $\mathrm{eV}$ by bare eye. The latter two exhibit a high spatial oscillation of positive and negative induced charge, alternating on neighboring atoms. However, all four figures show charge distributions with a nonvanishing dipole moment, in accordance with the employed dipolar coupling mechanism. As we will outline below, one has to be careful in deciding on the character of a resonance by only looking at the induced charge distribution in real space. To achieve a more coherent picture of the population dynamics, we have to consider energy space as well.
Energy Space Dynamics. Figure 8 shows the same dynamics in energy space. We depict the occupation difference

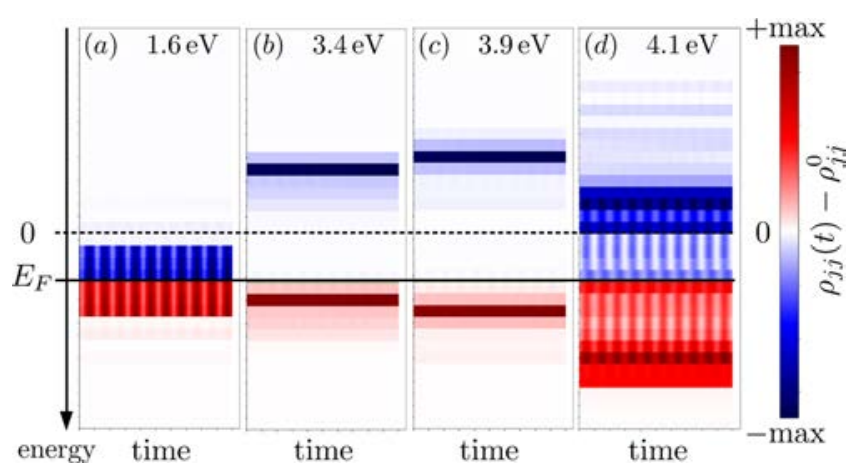

Figure 8. Population difference $\rho_{i j}(t)-\rho_{i j}^{0}$ of the energy states $\{|j\rangle\}$ of the dimer chain doped with 8 additional electrons under CW illumination for the last five optical cycles of the simulation period with respect to the ground state $\rho^{0}$. Initially, all the states below the Fermi energy are populated by 2 electrons, the states above are empty. The illumination frequencies coincide with those in Figure 6 and are chosen such that one of the resonances shows collective behavior, $\hbar \omega$ $=1.6 \mathrm{eV}$ in (a), two show single particle like behavior, $\hbar \omega=3.4 \mathrm{eV}$ in (b) and $\hbar \omega=3.9 \mathrm{eV}$ in (c), and one is of hybrid character, $\hbar \omega=4.1$ $\mathrm{eV}$ in (d).

$\rho_{j j}(t)-\rho_{j j}^{0}$ of the states near the Fermi energy with respect to the initial state for the last five optical cycles of the simulation period as a function of time in energy basis $\{|j\rangle\}$. The states below the Fermi energy are initially occupied by two electrons and lose occupation during $\mathrm{CW}$ illumination, indicated by the blue color. In contrast, the states above the Fermi energy, initially empty, gain occupation with time (red).

For the predominantly collective resonance at $\hbar \omega=1.6 \mathrm{eV}$ (a) and the hybrid one at $\hbar \omega=4.1 \mathrm{eV}$ (d), the occupation differences oscillate in time with frequency $\omega$, revealing rich dynamics of the electrons near the Fermi energy that is indicated in the plot by the horizontal solid line. This agrees to prior findings from Townsend and Bryant who called this behavior "sloshing" ${ }^{59,60}$ The oscillation pattern reveals a high symmetry with respect to the Fermi energy. The electron cloud collectively moves back and forth from below the Fermi energy to above it and back again in an oscillatory manner.

The predominantly single particle like resonances at $\hbar \omega=$ $3.4 \mathrm{eV}$ and $\hbar \omega=3.9 \mathrm{eV}$ behave qualitatively different. The most notable difference to the collective resonances is that the patterns, as shown in Figure 8 b,c, show a symmetry with respect to the $E=0$ line and not to the Fermi energy. This indicates that the set of active transitions is not determined by the number of doping electrons but purely by the electronic landscape and electrons from deep inside the Fermi sea can be lifted up to states far above the Fermi energy, as expected for single particle like electron-hole creation processes. The states involved in these resonances gain (lose) population in a Rabi flopping mechanism. For states below the Fermi energy, $\partial \rho_{i j}(t) / \partial t<0$ holds for all times of the simulation period, which is much shorter than the characteristic time scale of the Rabi flopping. Accordingly, for the states above the Fermi energy, we observe $\partial \rho_{j i}(t) / \partial t>0$, again in accordance with Townsend and Bryant who called this behavior "inver sion". ${ }^{59,60}$ Please note that this inversion process takes place on larger time scales than the optical cycles, and the full flopping cycle, therefore, cannot be seen in Figure 8. In the 
following, we want to introduce the EPI, which is capable of distinguishing these resonances based on energy space dynamics.

Construction of the EPI. In this section, with the EPI, we present a figure of merit that clearly distinguishes the single particle like resonances in a nanostructure from the plasmonic ones. Most suitable for the simulation method presented above is a measure based on the density matrix elements $\rho_{i j}$ of the antennas. However, we want to point out that the applicability of the EPI is not limited to density matrix based simulation schemes but can be also applied to wavefunction based methods like TDDFT that lack particle hole symmetry, for instance. One can retrieve the weight of the transitions which are involved in a resonance by the projections $\left\langle\varphi_{j}(t) \mid \varphi_{j^{\prime}}(0)\right\rangle$ of Kohn-Sham wave functions in TDDFT as well, ${ }^{58-60}$ being analogues of the coherences $\rho_{i j j^{\prime}}(t)$ used in this work and closely related to the transition contribution map. ${ }^{97}$

Single particle like transitions that occur between states $|j\rangle$ and $\left|j^{\prime}\right\rangle$ with energies $E_{j}$ and $E_{j^{\prime}}$ become apparent in the density matrix of the system as coherence elements $\rho_{i j^{\prime}}$, indicating said coupling. Therefore, we define the quantity $\tilde{\rho}^{\omega}$, which is large if such a coherence element exists between states whose energy difference matches the energy of the incident irradiation

$$
\tilde{\rho}_{j j^{\prime}}^{\omega}=\frac{\left|\rho_{j j^{\prime}}^{\omega}\right|}{\| E_{j}-E_{j^{\prime}}|-\hbar \omega+i \varepsilon|^{2}}
$$

Here, $\rho^{\omega}$ is the density matrix of the nanostructure in the energy basis after it has been exposed to $\mathrm{CW}$ illumination at frequency $\omega$ for a sufficiently long time (several tens of optical cycles at least). We introduced $\varepsilon=0.05 \mathrm{eV}$ which assures the regularity of the denominator, even if the incident light hits a transition frequency perfectly. It is in the order of the scattering time of electrons in bulk graphene ${ }^{94}$ used in eq 5. For a detailed discussion on the dependence of the EPI on $\varepsilon$, see Figure $S 1$ in the Supporting Information. Please note again, the matrix $\rho^{\omega}$ has been depleted on its diagonal before entering the definition of $\tilde{\rho}^{\omega}$ in eq 6 , that is, $\rho^{\omega} \rightarrow \rho^{\omega}-\sum_{j} \rho_{j j}^{\omega}|j\rangle\langle j|$. The quantity in eq 6 enhances transitions that meet the energy matching conditions $\left|E_{j}-E_{j^{\prime}}\right| \approx \hbar \omega$ and disregards elements in $\rho^{\omega}$ that are of plasmonic nature and, therefore, cannot be related to single particle transitions in the energy landscape of the structure. Please note that the quantity in eq 6 is closely related to the frequency dependent noninteracting suscepti bility of the random phase approximation framework

$$
\chi_{l l^{\prime}}^{0}(\omega)=\frac{2 e^{2}}{\hbar} \sum_{j j^{\prime}}\left(f_{j}-f_{j^{\prime}}\right) \frac{a_{j l} a_{j l^{\prime}}^{*} a_{j^{\prime} l}^{*} a_{j^{\prime} l^{\prime}}}{\left(E_{j}-E_{j^{\prime}}\right)-\hbar(\omega+i /(2 \tau))}
$$

which also exhibits poles at spectral positions that can be attributed to single particle like transitions. ${ }^{41,62}$

In the TB based simulation scheme employed in this work, one can additionally exploit the particle hole symmetry of the energy landscape in moderately doped systems by employing

$$
\tilde{\rho}_{j j^{\prime}}^{\omega}=\frac{\left|\rho_{j j^{\prime}}^{\omega} \rho_{N-j^{\prime}-1, N-j-1}^{\omega}\right|}{\| E_{j}-E_{j^{\prime}}|-\hbar \omega+i \varepsilon|^{2}}
$$

instead of the more generally applicable expression in eq 6 . Here, moderately doped means that the Fermi energy in the system is non zero but smaller in the absolute value than half the energy carried by a single photon of the incident electric pulse, that is, $0<\left|E_{\mathrm{F}}\right|<\hbar \omega / 2$. In this case, single particle like transitions that meet the energy matching conditions are not Pauli blocked and appear symmetrically with respect to the blue antidiagonal of the density matrix. The GNAs under consideration below are built of several hundreds of atoms and only a small number of doping electrons are needed to efficiently launch plasmons. ${ }^{41}$ Consequently, the GNAs meet this constraint very well. In the expression for $\tilde{\rho}^{\omega}$ given in eq 7 , transitions not occurring symmetrically to the antidiagonal of $\rho^{\omega}$ (blue diagonal dashed line in Figures 4-6) are suppressed and cannot be found in $\tilde{\rho}^{\omega}$.

As a result, for a single particle like resonance, we expect that $\tilde{\rho}^{\omega}$ is nearly proportional to $\rho^{\omega}$, whereas for plasmonic resonances, the two matrices differ substantially from each other. To measure this similarity, we define a scalar product of two matrices $a$ and $b$ according to

$$
\langle a, b\rangle:=\frac{\sum_{\mathrm{mn}}\left|a_{\mathrm{mn}} b_{\mathrm{mn}}\right|}{\left(\sum_{\mathrm{mn}}\left|a_{\mathrm{mn}}\right|^{2} \cdot \sum_{\mathrm{mn}}\left|b_{\mathrm{mn}}\right|^{2}\right)^{1 / 2}} \in[0,1]
$$

We emphasize that the matrices $a$ and $b$ do not necessarily need to be normalized in any sense for this scalar product to work as a measure of similarity. Indeed, it can be understood as a measure of how similar the magnitude of the elements is distributed and disseminated in $a$ and $b$, and not of how large the elements are in absolute value. As a consequence, our figure of merit is both independent of time and independent of the strength of the resonance in the absorption cross section. Finally, we define the EPI as

$$
\operatorname{EPI}(\omega)=1-\left\langle\tilde{\rho}^{\omega}, \rho^{\omega}\right\rangle \in[0,1]
$$

which is a measure of how strongly the resonance is built up by plasmonic contributions, that is, how strongly the amplification of the single particle like transitions affects the elements of $\rho^{\omega}$. If the resonance is purely single particle like, then by applying eqs 6 or 7 , we obtain a rescaled version of $\rho^{\omega}$, the scalar product $\left\langle\tilde{\rho}^{\omega}, \rho^{\omega}\right\rangle$ is close to unity, and $\operatorname{EPI}(\omega)=1-\left\langle\tilde{\rho}^{\omega}, \rho^{\omega}\right\rangle \approx$ 0 . On the other hand, if the resonance is plasmonic and built up by transitions around the Fermi energy that do not meet $\left|E_{j}-E_{j^{\prime}}\right| \approx \hbar \omega$, then $\operatorname{EPI}(\omega) \approx 1$, unless the involved transitions accidently both meet the energy matching condition and are situated symmetrically around $E=0$. We want to emphasize that the EPI is independent of the point in time that is chosen to pick $\rho^{\omega}$. Even though the elements of $\rho^{\omega}$ might change with time, the EPI itself does not because it is independent of the absolute values of $\rho_{j j^{\prime}}^{\omega}$. For the same reason, the EPI is independent of the relative height of the resonance in the absorption spectrum. The EPI can be applied to all the resonances in the spectrum, that is, whenever the structure shows an optical response.

Figure 9 shows the EPI (purple diamond elements), applied to various resonances of the linear (left column) and dimer chain (right column) in the undoped case (top row) and in the case of 8 doping electrons (bottom row) alongside with their absorption spectra (black solid lines). As outlined above, the linear chain's absorption spectrum is dominated by plasmonic resonances. However, we see that the EPI for the undoped linear chain, as shown in Figure 9a, is spread across almost the whole interval $[0,1]$. This is because the energy mesh provided by the TB method to construct the density matrix is not dense enough to resolve the energy region around the Fermi energy sufficiently well. Especially for small irradiation frequencies, the plasmonic contributions in $\rho^{\omega}$ have a nonvanishing overlap 

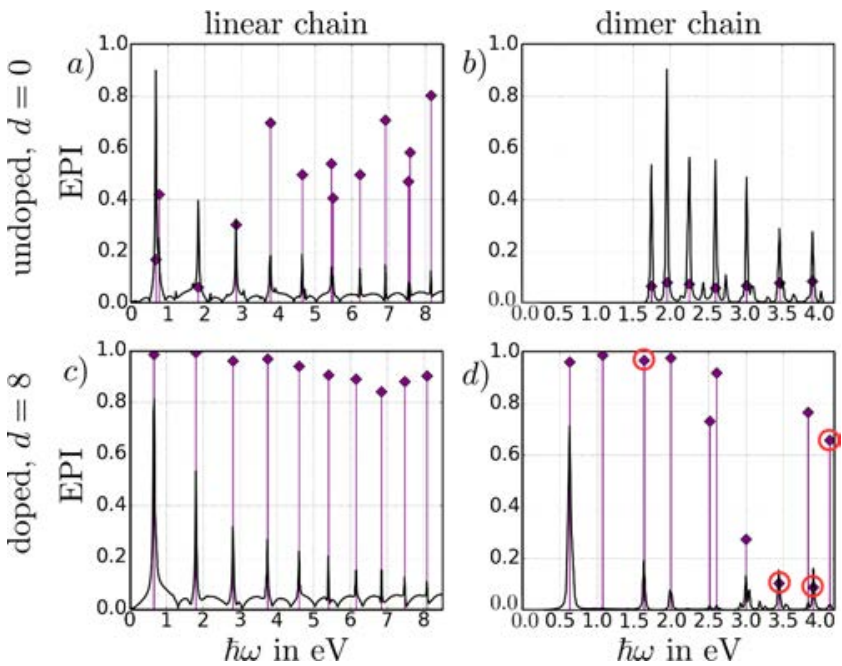

Figure 9. EPI (purple diamond elements), evaluated for the linear chain in the undoped case (a), with 8 doping electrons (c), and for the dimer chain in the undoped case (b) and with 8 doping electrons (d) alongside the absorption cross sections (black solid lines). The red circles mark the resonances investigated in Figures 6-8 in more detail. Please note that the energy axis for the linear chain ranges up to $9 \mathrm{eV}$, whereas the dimer chain is shown for energies up to $4.2 \mathrm{eV}$. The upper bound was chosen such that comparably many resonances could be investigated in both structures.

with their scaled correspondences in $\tilde{\rho}^{\omega}$. Also, the system is not doped and both the plasmonic and the single particle like transitions lie symmetric with respect to the antidiagonal of $\rho^{\omega}$. This misinterpretation does not occur in doped systems (see Figure 9c) and in systems that are large enough such that the TB method provides enough single particle energy states to resolve the energy landscape sufficiently well (see the hexagonal GNA below). Alternatively, to overcome this limitation of the applicability of the EPI to TB based simulations, one can employ simulation techniques that provide more energy states per atom, for example TDDFT.

This problem does not exist for the 8 fold doped linear chain, as shown in Figure 9c, because of the asymmetry of the transitions contributing to all resonances with respect to the antidiagonal of $\rho^{\omega}$. The EPI is well above 0.8 for all the investigated resonances, classified therefore as plasmonic in agreement with the qualitative discussion of the linear chain. In real space, the induced charge oscillation pattern exhibits an odd number of nodes, corresponding to dipole allowed plasmonic modes (not shown).

The pronounced resonances of the undoped dimer chain, as shown in Figure 9b, exhibit an EPI well below 0.1. The resonances are purely built up by single particle like transitions which meet the energy matching conditions. Consequently, all the resonances are classified single particle like in agreement with the qualitative discussion above.

The 8 fold doped dimer chain, as shown in Figure 9d, is the most complex and interesting structure under investigation. It supports single particle like resonances, plasmonic resonances, and hybrid ones. Those that show predominantly single particle like behavior have an EPI well below 0.3, two of which at $\hbar \omega=3.4 \mathrm{eV}$ and $\hbar \omega=3.9 \mathrm{eV}$, we already investigated in more detail in Figures $6-8$. They are highlighted by red circles. The remaining two circles around the resonances with EPI around 0.95 (almost purely plasmonic) and 0.65 (hybrid), highlight the resonances at $\hbar \omega=1.6 \mathrm{eV}$ and $\hbar \omega=4.1 \mathrm{eV}$, investigated in Figures 6-8 as well. In fact, by having a look at the population difference of the resonance around $1.6 \mathrm{eV}$, as shown in Figure $8 \mathrm{a}$, we see that it is purely built up by collectively oscillating transitions. In contrast, the resonance around $4.1 \mathrm{eV}$, as shown in Figure $8 \mathrm{~d}$, shows both the oscillatory behavior of plasmonic transitions and monotoni cally increasing populations indicating single particle like transitions. That renders this resonance hybrid in the sense that both mechanisms contribute substantially and leads to an intermediate EPI of 0.65 . We want to emphasize that this qualitative difference between the resonances at 1.6 and $4.1 \mathrm{eV}$ is not revealed by the real space oscillation patterns of induced charges, as shown in Figure 7a,d. They both look collective and plasmon like. We, therefore, conclude that further resonance classification efforts should predominantly rely on energy space considerations. Neglecting electron-electron interaction in the simulation, as it was done in the scaling approach of Bernadotte et al., ${ }^{62}$ results in a different spectrum with resonances of predominantly single particle like nature (see Figure S2 in the Supporting Information). Figure S3 of the Supporting Information shows the absorption cross sections of the 8 fold doped linear and dimer chains as a function of the Coulomb interaction strength. The scaling approach and the EPI are in perfect agreement in identifying the plasmonic resonances. However, the number of simulations needed to draw reliable conclusions from the scaling approach is much higher than for the EPI method. In the scaling approach, it is required to compare results for different Coulomb interaction strengths, whereas when using the EPI, a single simulation suffices to assign the EPI to the interval $[0,1]$. Furthermore, the scaling approach allows for a binary conclusion only (shifting or nonshifting as a function of Coulomb interaction strength), whereas the EPI measures to what extent hybrid resonances are single particle like and plasmonic. To demon strate that the EPI provides reliable results also in the regime where plasmonic and single particle like resonances overlap spectrally and the energy of plasmonic modes exceed the HOMO-LUMO gap, we discuss the 20 fold doped dimer chain in the Supporting Information (see Figure S4).

Concluding this section, we want to stress that the immunity of a resonance in the absorption spectrum against a change in doping does not immediately reveal its single particle like nature. The property is necessary but not sufficient to draw this conclusion. We can see in Figure $3 a$ that all the resonances of the linear chain are insensitive to doping although they are of plasmonic nature. Conversely, it is also not correct that all plasmons blue shift with increasing doping as can be appreciated from the very same figure. A plasmonic resonance undergoes a spectral shift while the doping changes, only if the local energy landscape environment around the Fermi energy changes as well. For an investigation of the resonances in a polyacene chain, as shown Figures S5 and S6 of the Supporting Information. In the following of the main paper, we want to apply the EPI to a physical structure already widely investigated, a hexagonal GNA.

Hexagonal GNA. The promising optical properties of GNAs of various shapes in tailored photonic environments have been investigated in many previous works. $^{13,40,41,73,76,77,79-81}$ They are believed to constitute an excellent platform for plasmon assisted nonlinear energy conversion processes. This renders them an optimal application to the EPI because it is of immense interest to find their plasmonic resonances. We want to explicitly pick out 
those resonances of the GNA's spectrum that have collective character and may provide all the beneficial effects that come along with a plasmonic response.

Figure 10a shows the absorption cross section of an armchair edged hexagonal GNA with 366 atoms for a number

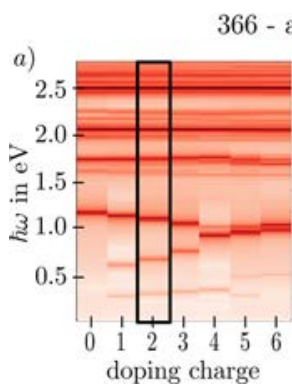

366 - atomic armchair hexagon

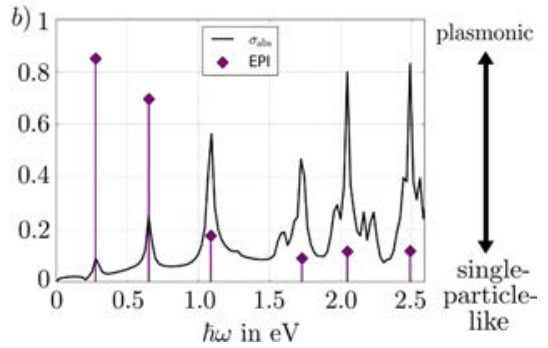

Figure 10. (a) Absorption cross section for the graphene armchair hexagon with 366 atoms as a function of doping charge. (b) EPI (purple diamond elements) for the resonances of the hexagon with 2 doping electrons alongside the absorption cross section (black solid line).

of doping electrons between 0 and 6 . We see that the system behaves similar to the dimer chain. This is because the energy landscapes of both structures are similar as well, exhibiting an energy gap across $E=0$. The GNA's spectrum has no low energy resonances in the undoped case. However, when additional electrons are introduced into the system, blue shifting low energy resonances start to appear. Simultaneously, like in Figure 3b, we notice the red shift of the lowest resonance that is present in the undoped case.

Real Space Dynamics. First, we investigate the real space dynamics of the induced charge on the flake. Figure 11 shows a

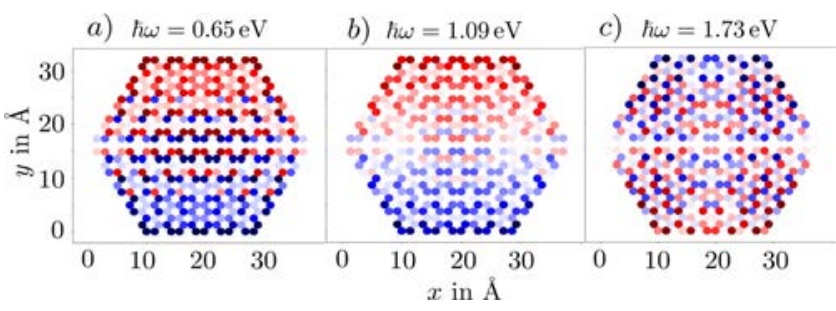

Figure 11. Snapshots of the induced charge distribution on the hexagonal GNA with 366 atoms and two doping electrons. The snapshots are taken at the time of maximum dipole moment under $y$ polarized CW illumination at the resonance frequencies (a) $\hbar \omega=$ $0.65 \mathrm{eV}$, (b) $\hbar \omega=1.09 \mathrm{eV}$, and (c) $\hbar \omega=1.73 \mathrm{eV}$.

snapshot of the induced charge on the hexagonal GNA with two doping electrons for three different CW illumination frequencies at the time of maximum dipole moment. The charge distributions for the illumination frequencies $\hbar \omega=0.65$ $\mathrm{eV}$ and $\hbar \omega=1.09 \mathrm{eV}$, as shown in Figure 11a,b, respectively, show a clear collective character. In both cases, we find a charge separation along a horizontal line intersecting the GNA through the middle and antinodes of the charge distribution at the top and bottom edge of the flake. A different pattern reveals the charge distribution of the resonance at $\hbar \omega=1.73$ $\mathrm{eV}$, as shown in Figure 11c. Similarly to the charge distribution patterns, as shown in Figure $7 b, c$, the spatial frequency of the charge distribution is much higher. From these considerations only, one could conclude that, just like for the dimer chain, the resonances with collective real space dynamics $(\hbar \omega=0.65 \mathrm{eV}$ and $\hbar \omega=1.09 \mathrm{eV}$ ) can be classified as plasmonic and the one exhibiting the more erratic pattern $(\hbar \omega=1.73 \mathrm{eV})$ single particle like. However, we will see below that also for this structure relying on real space dynamics only lets us draw wrong conclusions.

Energy Space Dynamics. We examine the very same three resonances in energy space and look for the fingerprints of plasmonic and single particle like transitions as introduced above for the dimer chain. Figure 12 shows the occupation

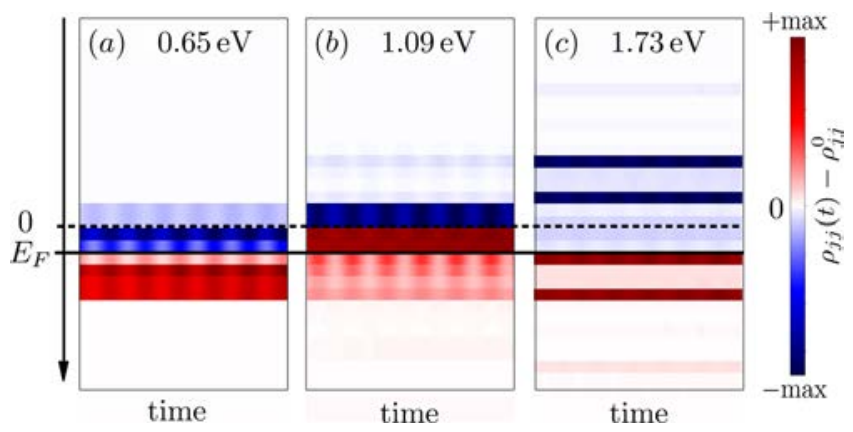

Figure 12. Population difference $\rho_{i j}(t)-\rho_{i j}^{0}$ of the energy states $\{|j\rangle\}$ of the hexagonal GNA doped with 2 electrons under $\mathrm{CW}$ illumination for the last five optical cycles of the simulation period with respect to the ground state $\rho^{0}$. The illumination frequencies coincide with those in Figure 11, $\hbar \omega=0.65 \mathrm{eV}(\mathrm{a}), \hbar \omega=1.09 \mathrm{eV}(\mathrm{b})$, and $\hbar \omega=1.73 \mathrm{eV}$ (c).

difference $\rho_{i j}(t)-\rho_{i j}^{0}$ of the GNA's energy states $\{|j\rangle\}$ with respect to the initial state $\rho^{0}$ near the Fermi energy for the last five optical cycles of the simulation period. The involved energy states of the resonance at $\hbar \omega=0.65 \mathrm{eV}$ (Figure 12a) lie mostly symmetrically to the Fermi energy and exhibit clear sloshing behavior both above and below $E_{\mathrm{F}}$. This reveals that they dynamically exchange population back and forth across the Fermi energy level, which is typical for plasmonic excitations. However, from the different color shadings, we can see that there is also a monotonically increasing (decreasing) component overlayed in the dynamics. Although this is generally true for all the resonances under consideration here, at the end of the day, the symmetry around $E_{\mathrm{F}}$ renders this resonance predominantly plasmonic.

In contrast, both the resonance at $\hbar \omega=1.09 \mathrm{eV}$ and $\hbar \omega=$ $1.73 \mathrm{eV}$ are built up by occupation dynamics in states that lie symmetrically to $E=0$ and that are mostly monotonically increasing or decreasing, revealing the "inversion" signature. The sloshing character of the population dynamics is less pronounced in both figures but not completely absent. Thus, from energy space considerations only, one could conclude that only the resonance at $\hbar \omega=0.65 \mathrm{eV}$ is of predominantly plasmonic nature, and the other two are predominantly single particle like. Please note that this contradicts the expectation that followed from real space considerations.

EPI for the GNA. To apply the EPI to the GNA, we investigate the six most prominent absorption peaks for $d=2$, as shown in Figure $10 \mathrm{~b}$. We find that the two resonances lowest in energy can be classified to be predominantly plasmonic with an EPI around 0.83 and 0.68 , respectively. This is expected because we cannot find these resonances in the undoped structure. They appear when at least one state above $E=0$ is populated and they blue shift with increasing doping. These are both clear signs for collective plasmon like behavior. The three resonances highest in energy with an EPI 
around 0.1 can be classified as predominantly single particle like. They do not exhibit any shift with doping and are therefore determined by the electronic structure of the GNA only. The resonance at $1.09 \mathrm{eV}$ is evaluated close to 0.2 . We can see that this latter resonance already exists in the undoped structure and can be attributed to a single particle transition. However, we also see that the resonance red shifts with increasing doping, which is a sign of collective behavior. At the end of the day, one can classify it as hybrid yet with a very strong single particle like contribution. This is in accordance with our expectation that followed from the energy space dynamics considerations in the previous section, cf. Figure 12, but does not coincide with the expectations that followed from real space dynamics of induced charge on the flake. For this reason, we have suggested a measure classifying the nature of a resonance in a nanoparticle based on energy space population dynamics, rather than on real space dynamics, as it was done by constructing the (generalized) plasmonicity index (G) PI. ${ }^{66,98}$ The GPI and the EPI should be seen as complementary. We want to point out here that our measure does not give information on how the resonance manifests itself in real space (like the PI and the GPI do). It concerns only the physical origin of the resonance. In other words, we probe if the resonance's existence can be explained by the energy landscape of the structure or if interaction energy has to be taken into account to explain its emergence.

\section{CONCLUSIONS}

In this paper, we have contributed to answering the question of what a plasmon in truly nanoscopic systems is. We have introduced an energy space based figure of merit for molecular structures in TB description which can also be applied in wave function based methods like TDDFT. It can tell apart resonances built up by single particle like transitions from those that reveal plasmonic behavior in the sense that their spectral position can only be explained by taking into account the interaction energy of electrons. The performance of the EPI was checked against both energy space and real space dynamics of the induced charge in the system. We obtain excellent agreement between our classification conclusions based on energy space investigations by bare eye on the one hand, and the EPI based on the antenna's density matrix elements on the other hand. Moreover, we are in excellent agreement with the scaling approach by Bernadotte et. al., ${ }^{62}$ which also relies on interaction energy considerations. However, using the EPI reduces the computational cost considerably. One single simulation rather than a full cycle of simulations with different interaction energy strengths is sufficient to characterize a resonance. We have shown that the real space oscillation patterns of hybrid resonances mostly resemble those of the plasmonic kind and miss single particle like characteristics. This renders real space unreliable in deciding the resonance's nature. One should rather rely on energy space dynamics in the first place, which exhibit clear signatures of both transition species simultaneously. Plasmonic transitions build up symmetrically with respect to the Fermi energy, and their population differences show rich oscillation dynamics in energy space ("sloshing"). Contrarily, single particle like transitions build up symmetrically with respect to the particle hole symmetry level, which is $E=0$ in the TB method introduced above. Their population differences increase (decrease) for states below (above) the Fermi energy in a Rabi flopping process ("inversion"). Furthermore, the immunity of a resonance's spectral position with changing doping is not a sufficient feature to conclude that it is of the single particle like nature. Conversely, some predominantly single particle like resonances may yet have a significant collective component responsible for spectral dependence on the doping level and dominating the real space charge distribution.

\section{AUTHOR INFORMATION}

\section{Corresponding Authors}

Marvin M. Müller - Institute of Theoretical Solid State Physics, Karlsruhe Institute of Technology (KIT), 76131 Karlsruhe, Germany;

Email: marvin.mueller@kit.edu

Miriam Kosik - Institute of Physics, Nicolaus Copernicus University in Torún, 87100 Torun, Poland; Email: mkosik@ doktorant.umk.pl

\section{Authors}

Marta Pelc - Donostia International Physics Center (DIPC), 20018 Donostia San Sebastián, Spain; Centro de Física de Materiales, CFM MPC CSIC UPV/EHU, 20018 Donostia San Sebastián, Spain

Garnett W. Bryant - Joint Quantum Institute, University of Maryland and National Institute of Standards and Technology, College Park, Maryland 20742, United States; Nanoscale Device Characterization Division, National Institute of Standards and Technology, Gaithersburg, Maryland 20899, United States

Andrés Ayuela - Donostia International Physics Center (DIPC), 20018 Donostia San Sebastián, Spain; Centro de Física de Materiales, CFM MPC CSIC UPV/EHU, 20018 Donostia San Sebastián, Spain

Carsten Rockstuhl - Institute of Theoretical Solid State Physics, Karlsruhe Institute of Technology (KIT), 76131 Karlsruhe, Germany; Institute of Nanotechnology, Karlsruhe Institute of Technology, 76021 Karlsruhe, Germany

Karolina Słowik - Institute of Physics, Nicolaus Copernicus University in Torú, 87100 Torun, Poland;

\section{Notes}

The authors declare no competing financial interest.

\section{ACKNOWLEDGMENTS}

M.M.M. acknowledges financial support through the Research Travel Grant by the Karlsruhe House of Young Scientists (KHYS). M.M.M. and C.R. acknowledge support by the 
Deutsche Forschungsgemeinschaft (DFG, German Research Foundation)-project number 378579271-within project RO 3640/8 1 and from the VolkswagenStiftung. M.M.M. is grateful for the support of the Torun Astrophysics/Physics Summer Program TAPS 2019 and the PROM project no. PPI/ PRO/2018/1/00016/U/001 by the Polish National Agency for Academic Exchange. M.M.M., M.K., and K.S. acknowledge the hospitality of the Donostia International Physics Center. M.K. acknowledges the financial support from the Foundation for Polish Science (FNP) (project First Team/2017 3/20 cofinanced by the European Union under the European Regional Development Fund). M.P. and A.A. acknowledge financial support by project PID2019 105488GB I00 of the Spanish Ministry of Science and Innovation, and the Gobierno Vasco-UPV/EHU project IT1246 19. K.S. acknowledges support from the National Science Centre, Poland (project no. 2016/23/G/ST3/0404).

\section{REFERENCES}

(1) Boltasseva, A.; Atwater, H. A. Low Loss Plasmonic Metamate rials. Science 2011, 331, 290-291.

(2) Maier, S. A. Plasmonics: Fundamentals and Applications; Springer Science \& Business Media, 2007.

(3) Zouhdi, S.; Sihvola, A.; Vinogradov, A. P. Metamaterials and Plasmonics: Fundamentals, Modelling, Applications; Springer Science \& Business Media, 2008.

(4) Cazalilla, M. A.; Dolado, J. S.; Rubio, A.; Echenique, P. M. Plasmonic excitations in noble metals: The case of Ag. Phys. Rev. B: Condens. Matter Mater. Phys. 2000, 61, 8033-8042.

(5) Pelton, M.; Aizpurua, J.; Bryant, G. Metal nanoparticle plasmonics. Laser Photonics Rev. 2008, 2, 136-159.

(6) Giannini, V.; Fernández Domínguez, A. I.; Heck, S. C.; Maier, S. A. Plasmonic nanoantennas: fundamentals and their use in controlling the radiative properties of nanoemitters. Chem. Rev. 2011, 111, 38883912.

(7) Koppens, F. H. L.; Chang, D. E.; García de Abajo, F. J. Graphene plasmonics: a platform for strong light-matter interactions. Nano Lett. 2011, 11, 3370-3377.

(8) Takahara, J.; Yamagishi, S.; Taki, H.; Morimoto, A.; Kobayashi, $\mathrm{T}$. Guiding of a one dimensional optical beam with nanometer diameter. Opt. Lett. 1997, 22, 475-477.

(9) Gramotnev, D. K.; Bozhevolnyi, S. I. Plasmonics beyond the diffraction limit. Nat. Photonics 2010, 4, 83.

(10) Novotny, L.; Hecht, B. Principles of Nano Optics; Cambridge University Press, 2012.

(11) Mikhailov, S. A. Theory of the giant plasmon enhanced second harmonic generation in graphene and semiconductor two dimensional electron systems. Phys. Rev. B: Condens. Matter Mater. Phys. 2011, 84, 045432.

(12) Ginzburg, P.; Krasavin, A.; Sonnefraud, Y.; Murphy, A.; Pollard, R. J.; Maier, S. A.; Zayats, A. V. Nonlinearly coupled localized plasmon resonances: Resonant second harmonic generation. Phys. Rev. B: Condens. Matter Mater. Phys. 2012, 86, 085422.

(13) Cox, J. D.; Marini, A.; García de Abajo, F. J. Plasmon assisted high harmonic generation in graphene. Nat. Commun. 2017, 8, 14380.

(14) Melendez, J.; Carr, R.; Bartholomew, D. U.; Kukanskis, K.; Elkind, J.; Yee, S.; Furlong, C.; Woodbury, R. A commercial solution for surface plasmon sensing. Sens. Actuators, B 1996, 35, 212-216.

(15) Awazu, K.; Rockstuhl, C.; Fujimaki, M.; Fukuda, N.; Tominaga, J.; Komatsubara, T.; Ikeda, T.; Ohki, Y. High sensitivity sensors made of perforated waveguides. Opt. Express 2007, 15, 2592-2597.

(16) Lee, C.; Dieleman, F.; Lee, J.; Rockstuhl, C.; Maier, S. A.; Tame, M. Quantum plasmonic sensing: beyond the shot noise and diffraction limit. ACS Photonics 2016, 3, 992-999.

(17) Hess, O.; Pendry, J. B.; Maier, S. A.; Oulton, R. F.; Hamm, J. M.; Tsakmakidis, K. L. Active nanoplasmonic metamaterials. Nat. Mater. 2012, 11, 573-584.
(18) Mühlig, S.; Cunningham, A.; Dintinger, J.; Scharf, T.; Bürgi, T.; Lederer, F.; Rockstuhl, C. Self assembled plasmonic metamaterials. Nanophot 2013, 2, 211-240.

(19) Koenderink, A. F. Plasmon nanoparticle array waveguides for single photon and single plasmon sources. Nano Lett. 2009, 9, 42284233.

(20) Chen, Y.; Lodahl, P.; Koenderink, A. F. Dynamically reconfigurable directionality of plasmon based single photon sources. Phys. Rev. B: Condens. Matter Mater. Phys. 2010, 82, No. 081402(R).

(21) Yu, P.; Wu, J.; Ashalley, E.; Govorov, A.; Wang, Z. Dual band absorber for multispectral plasmon enhanced infrared photodetection. J. Phys. D: Appl. Phys. 2016, 49, 365101.

(22) Brongersma, M. L.; Halas, N. J.; Nordlander, P. Plasmon induced hot carrier science and technology. Nat. Nanotechnol. 2015, $10,25$.

(23) Kim, J. T.; Yu, Y. J.; Choi, H.; Choi, C. G. Graphene based plasmonic photodetector for photonic integrated circuits. Opt. Express 2014, 22, 803-808.

(24) Salamin, Y.; Ma, P.; Baeuerle, B.; Emboras, A.; Fedoryshyn, Y.; Heni, W.; Cheng, B.; Josten, A.; Leuthold, J. $100 \mathrm{GHz}$ plasmonic photodetector. ACS Photonics 2018, 5, 3291-3297.

(25) Mousavi, S. S.; Stöhr, A.; Berini, P. Plasmonic photodetector with terahertz electrical bandwidth. Appl. Phys. Lett. 2014, 104, 143112.

(26) Patrick O’Neal, D.; Hirsch, L. R.; Halas, N. J.; Payne, J. D.; West, J. L. Photo thermal tumor ablation in mice using near infrared absorbing nanoparticles. Cancer Lett. 2004, 209, 171-176.

(27) Qian, X.; Peng, X. H.; Ansari, D. O.; Yin Goen, Q.; Chen, G. Z.; Shin, D. M.; Yang, L.; Young, A. N.; Wang, M. D.; Nie, S. In vivo tumor targeting and spectroscopic detection with surface enhanced Raman nanoparticle tags. Nat. Biotechnol. 2008, 26, 83-90.

(28) Hirsch, L. R.; Stafford, R. J.; Bankson, J. A.; Sershen, S. R.; Rivera, B.; Price, R. E.; Hazle, J. D.; Halas, N. J.; West, J. L. Nanoshell mediated near infrared thermal therapy of tumors under magnetic resonance guidance. Proc. Natl. Acad. Sci. U.S.A. 2003, 100, 1354913554.

(29) Novoselov, K. S.; Geim, A. K.; Morozov, S. V.; Jiang, D.; Zhang, Y.; Dubonos, S. V.; Grigorieva, I. V.; Firsov, A. A. Electric field effect in atomically thin carbon films. Science 2004, 306, 666-669.

(30) Bozhevolnyi, S. I.; Mortensen, N. A. Plasmonics for emerging quantum technologies. Nanophot 2017, 6, 1185-1188.

(31) Bryant, G. W.; Waks, E.; Krenn, J. R. Plasmonics: The rise of quantum effects. Opt. Photonics News 2014, 25, 50-53.

(32) Tame, M. S.; McEnery, K. R.; Özdemir, Ş. K.; Lee, J.; Maier, S. A.; Kim, M. S. Quantum plasmonics. Nat. Phys. 2013, 9, 329-340.

(33) Wang, F.; Shen, Y. R. General properties of local plasmons in metal nanostructures. Phys. Rev. Lett. 2006, 97, 206806.

(34) Wubs, M.; Mortensen, N. A. Quantum Plasmonics; Springer, 2017; pp 279-302.

(35) Chang, D. E.; Sørensen, A. S.; Hemmer, P.; Lukin, M. Quantum optics with surface plasmons. Phys. Rev. Lett. 2006, 97, 053002 .

(36) Akimov, A. V.; Mukherjee, A.; Yu, C. L.; Chang, D. E.; Zibrov, A. S.; Hemmer, P. R.; Park, H.; Lukin, M. D. Generation of single optical plasmons in metallic nanowires coupled to quantum dots. Nature 2007, 450, 402-406.

(37) Kühn, S.; Håkanson, U.; Rogobete, L.; Sandoghdar, V. Enhancement of single molecule fluorescence using a gold nano particle as an optical nanoantenna. Phys. Rev. Lett. 2006, 97, 017402.

(38) Kolesov, R.; Grotz, B.; Balasubramanian, G.; Stöhr, R. J.; Nicolet, A. A. L.; Hemmer, P. R.; Jelezko, F.; Wrachtrup, J. Wave particle duality of single surface plasmon polaritons. Nat. Phys. 2009, $5,470-474$.

(39) Purcell, E. M.; Torrey, H. C.; Pound, R. V. Resonance absorption by nuclear magnetic moments in a solid. Phys. Rev. 1946, 69, 37.

(40) Thongrattanasiri, S.; Manjavacas, A.; García de Abajo, F. J. Quantum finite size effects in graphene plasmons. ACS Nano 2012, 6, $1766-1775$. 
(41) Manjavacas, A.; Thongrattanasiri, S.; de Abajo, F. J. G. Plasmons driven by single electrons in graphene nanoislands. Nanophot 2013, 2, 139-151.

(42) Recio, F. J.; Zabala, N.; Rivacoba, A.; Crespo, P.; Ayuela, A.; Echenique, P. M.; Hernando, A. Optical resonances of colloidal gold nanorods: from seeds to chemically thiolated long nanorods. J. Phys. Chem. C 2015, 119, 7856-7864.

(43) Sinha Roy, R.; García González, P.; Weissker, H. C.; Rabilloud, F.; Fernández Domínguez, A. I. Classical and ab initio plasmonics meet at sub nanometric noble metal rods. ACS Photonics 2017, 4, $1484-1493$.

(44) Giovannini, T.; Rosa, M.; Corni, S.; Cappelli, C. A classical picture of subnanometer junctions: an atomistic Drude approach to nanoplasmonics. Nanoscale 2019, 11, 6004-6015.

(45) Giovannini, T.; Bonatti, L.; Polini, M.; Cappelli, C. Graphene Plasmonics: Fully Atomistic Approach for Realistic Structures. J. Phys. Chem. Lett. 2020, 11, 7595-7602.

(46) Beck, D. E. Self consistent calculation of the eigenfrequencies for the electronic excitations in small jellium spheres. Phys. Rev. B: Condens. Matter Mater. Phys. 1987, 35, 7325.

(47) Reinhard, P. G.; Brack, M.; Genzken, O. Random phase approximation in a local representation. Phys. Rev. A 1990, 41, 5568

(48) Kümmel, S.; Andrae, K.; Reinhard, P. G. Collectivity in the optical response. of small metal clusters. Appl. Phys. B 2001, 73, 293297.

(49) Raitza, T.; Reinholz, H.; Reinhard, P. G.; Röpke, G.; Broda, I. Spatially resolved collective excitations of nano plasmas via molecular dynamics simulations and fluid dynamics. New J. Phys. 2012, 14, 115016.

(50) Guidez, E. B.; Aikens, C. M. Quantum mechanical origin of the plasmon: from molecular systems to nanoparticles. Nanoscale 2014, 6 , $11512-11527$.

(51) Guidez, E. B.; Aikens, C. M. Plasmon resonance analysis with configuration interaction. Phys. Chem. Chem. Phys. 2014, 16, 1550115509 .

(52) Guerrini, M.; Calzolari, A.; Varsano, D.; Corni, S. Quantifying the Plasmonic Character of Optical Excitations in a Molecular J Aggregate. J. Chem. Theory Comput. 2019, 15, 3197-3203.

(53) Liu, C.; Kloppenburg, J.; Yao, Y.; Ren, X.; Appel, H.; Kanai, Y.; Blum, V. All electron ab initio Bethe Salpeter equation approach to neutral excitations in molecules with numeric atom centered orbitals. J. Chem. Phys. 2020, 152, 044105.

(54) Blase, X.; Attaccalite, C. Charge transfer excitations in molecular donor acceptor complexes within the many body Bethe Salpeter approach. Appl. Phys. Lett. 2011, 99, 171909.

(55) Ekardt, W. Size dependent photoabsorption and photoemission of small metal particles. Phys. Rev. B: Condens. Matter Mater. Phys. 1985, 31, 6360 .

(56) Ekardt, W.; Penzar, Z. Collective excitations in open shell metal clusters: The time dependent local density approximation applied to the self consistent spheroidal jellium particle. Phys. Rev. B: Condens. Matter Mater. Phys. 1991, 43, 1322.

(57) Puska, M. J.; Nieminen, R. M.; Manninen, M. Electronic polarizability of small metal spheres. Phys. Rev. B: Condens. Matter Mater. Phys. 1985, 31, 3486.

(58) Townsend, E.; Bryant, G. W. Plasmonic properties of metallic nanoparticles: The effects of size quantization. Nano Lett. 2012, 12, 429-434.

(59) Townsend, E.; Bryant, G. W. Which resonances in small metallic nanoparticles are plasmonic? J. Opt. 2014, 16, 114022.

(60) Townsend, E.; Debrecht, A.; Bryant, G. W. Approaching the quantum limit for nanoplasmonics. J. Mater. Res. 2015, 30, 23892399.

(61) Krauter, C. M.; Bernadotte, S.; Jacob, C. R.; Pernpointner, M.; Dreuw, A. Identification of plasmons in molecules with scaled ab initio approaches. J. Phys. Chem. C 2015, 119, 24564-24573.

(62) Bernadotte, S.; Evers, F.; Jacob, C. R. Plasmons in molecules. J. Phys. Chem. C 2013, 117, 1863-1878.
(63) Piccini, G.; Havenith, R. W. A.; Broer, R.; Stener, M. Gold nanowires: a time dependent density functional assessment of plasmonic behavior. J. Phys. Chem. C 2013, 117, 17196-17204.

(64) Bursi, L.; Calzolari, A.; Corni, S.; Molinari, E. Light induced field enhancement in nanoscale systems from first principles: the case of polyacenes. ACS Photonics 2014, 1, 1049-1058.

(65) Gieseking, R. L. M.; Ashwell, A. P.; Ratner, M. A.; Schatz, G. C. Analytical Approaches To Identify Plasmon like Excited States in Bare and Ligand Protected Metal Nanoclusters. J. Phys. Chem. C 2020, 124, 3260-3269.

(66) Bursi, L.; Calzolari, A.; Corni, S.; Molinari, E. Quantifying the plasmonic character of optical excitations in nanostructures. ACS Photonics 2016, 3, 520-525.

(67) Noguchi, T.; Shimamoto, T.; Watanabe, K. Photoabsorption Spectra of Graphitic Nanostructures by Time Dependent Density Functional Theory. e J. Surf. Sci. Nanotechnol. 2005, 3, 439-443.

(68) Yan, J.; Yuan, Z.; Gao, S. End and central plasmon resonances in linear atomic chains. Phys. Rev. Lett. 2007, 98, 216602.

(69) Yan, J.; Gao, S. Plasmon resonances in linear atomic chains: free electron behavior and anisotropic screening of $\mathrm{d}$ electrons. Phys. Rev. B: Condens. Matter Mater. Phys. 2008, 78, 235413.

(70) Manjavacas, A.; Marchesin, F.; Thongrattanasiri, S.; Koval, P.; Nordlander, P.; Sánchez Portal, D.; García de Abajo, F. J. Tunable molecular plasmons in polycyclic aromatic hydrocarbons. ACS Nano 2013, 7, 3635-3643.

(71) Kuisma, M.; Sakko, A.; Rossi, T. P.; Larsen, A. H.; Enkovaara, J.; Lehtovaara, L.; Rantala, T. T. Localized surface plasmon resonance in silver nanoparticles: Atomistic first principles time dependent density functional theory calculations. Phys. Rev. B: Condens. Matter Mater. Phys. 2015, 91, 115431.

(72) Ezawa, M. Metallic graphene nanodisks: Electronic and magnetic properties. Phys. Rev. B: Condens. Matter Mater. Phys. 2007, 76, 245415.

(73) Güçlü, A. D.; Potasz, P.; Hawrylak, P. Excitonic absorption in gate controlled graphene quantum dots. Phys. Rev. B: Condens. Matter Mater. Phys. 2010, 82, 155445.

(74) Jaskólski, W.; Ayuela, A.; Pelc, M.; Santos, H.; Chico, L. Edge states and flat bands in graphene nanoribbons with arbitrary geometries. Phys. Rev. B: Condens. Matter Mater. Phys. 2011, 83, 235424.

(75) Potasz, P.; Güçlü, A.; Wójs, A.; Hawrylak, P. Electronic properties of gated triangular graphene quantum dots: Magnetism, correlations, and geometrical effects. Phys. Rev. B: Condens. Matter Mater. Phys. 2012, 85, 075431.

(76) Christensen, T.; Wang, W.; Jauho, A. P.; Wubs, M.; Mortensen, N. A. Classical and quantum plasmonics in graphene nanodisks: Role of edge states. Phys. Rev. B: Condens. Matter Mater. Phys. 2014, 90, No. 241414(R).

(77) Cox, J. D.; García de Abajo, F. J. Electrically tunable nonlinear plasmonics in graphene nanoislands. Nat. Commun. 2014, 5, 5725.

(78) Grigorenko, A. N.; Polini, M.; Novoselov, K. S. Graphene plasmonics. Nat. Photonics 2012, 6, 749.

(79) Cox, J. D.; García de Abajo, F. J. Plasmon enhanced nonlinear wave mixing in nanostructured graphene. ACS Photonics 2015, 2, $306-312$.

(80) Cox, J. D.; García de Abajo, F. J. Transient nonlinear plasmonics in nanostructured graphene. Optica 2018, 5, 429-433.

(81) Cox, J. D.; García de Abajo, F. J. Nonlinear Graphene Nanoplasmonics. Acc. Chem. Res. 2019, 52, 2536-2547.

(82) Chapkin, K. D.; Bursi, L.; Stec, G. J.; Lauchner, A.; Hogan, N. J.; Cui, Y.; Nordlander, P.; Halas, N. J. Lifetime dynamics of plasmons in the few atom limit. Proc. Natl. Acad. Sci. U.S.A. 2018, 115, 91349139.

(83) Chapkin, K. D.; Bursi, L.; Clark, B. D.; Wu, G.; Lauchner, A.; Tsai, A. L.; Nordlander, P.; Halas, N. J. Effects of Electronic Structure on Molecular Plasmon Dynamics. J. Phys. Chem. C 2020, 124, 2045020457. 
(84) Potasz, P.; Güçlü, A.; Hawrylak, P. Spin and electronic correlations in gated graphene quantum rings. Phys. Rev. B: Condens. Matter Mater. Phys. 2010, 82, 075425.

(85) Alonso Calafell, I.; Cox, J.; Radonjić, M.; Saavedra, J.; García de Abajo, F. J.; Rozema, L.; Walther, P. Quantum computing with graphene plasmons. npj Quantum Inf. 2019, 5, 93.

(86) Brolo, A. G. Plasmonics for future biosensors. Nat. Photonics 2012, 6, 709.

(87) Wagner, M.; Fei, Z.; McLeod, A. S.; Rodin, A. S.; Bao, W.; Iwinski, E. G.; Zhao, Z.; Goldflam, M.; Liu, M.; Dominguez, G.; et al. Ultrafast and nanoscale plasmonic phenomena in exfoliated graphene revealed by infrared pump-probe nanoscopy. Nano Lett. 2014, 14, 894-900.

(88) Ni, G. X.; Wang, L.; Goldflam, M. D.; Wagner, M.; Fei, Z.; McLeod, A. S.; Liu, M. K.; Keilmann, F.; Özyilmaz, B.; Castro Neto, A. H.; et al. Ultrafast optical switching of infrared plasmon polaritons in high mobility graphene. Nat. Photonics 2016, 10, 244-247.

(89) Johannsen, J. C.; Ulstrup, S.; Cilento, F.; Crepaldi, A.; Zacchigna, M.; Cacho, C.; Turcu, I. E.; Springate, E.; Fromm, F.; Raidel, C.; et al. Direct view of hot carrier dynamics in graphene. Phys. Rev. Lett. 2013, 111, 027403.

(90) Gierz, I.; Petersen, J. C.; Mitrano, M.; Cacho, C.; Turcu, I. C. E.; Springate, E.; Stöhr, A.; Köhler, A.; Starke, U.; Cavalleri, A. Snapshots of non equilibrium Dirac carrier distributions in graphene. Nat. Mater. 2013, 12, 1119-1124.

(91) Breusing, M.; Kuehn, S.; Winzer, T.; Malić, E.; Milde, F.; Severin, N.; Rabe, J.; Ropers, C.; Knorr, A.; Elsaesser, T. Ultrafast nonequilibrium carrier dynamics in a single graphene layer. Phys. Rev. B: Condens. Matter Mater. Phys. 2011, 83, 153410.

(92) Pelc, M.; Chico, L.; Ayuela, A.; Jaskólski, W. Grain boundaries with octagonal defects in graphene nanoribbons and nanotubes. Phys. Rev. B: Condens. Matter Mater. Phys. 2013, 87, 165427.

(93) Castro Neto, A. H.; Guinea, F.; Peres, N. M.; Novoselov, K. S.; Geim, A. K. The electronic properties of graphene. Rev. Mod. Phys. 2009, 81, 109.

(94) Novoselov, K. S.; Geim, A. K.; Morozov, S. V.; Jiang, D.; Katsnelson, M. I.; Grigorieva, I. V.; Dubonos, S. V.; Firsov, A. A. Two dimensional gas of massless Dirac fermions in graphene. Nature 2005, 438, 197-200.

(95) Fölsch, S.; Hyldgaard, P.; Koch, R.; Ploog, K. Quantum confinement in monatomic $\mathrm{Cu}$ chains on $\mathrm{Cu}$ (111). Phys. Rev. Lett. 2004, 92, 056803.

(96) de Vega, S.; Cox, J. D.; Sols, F.; García de Abajo, F. J. Strong field driven dynamics and high harmonic generation in interacting one dimensional systems. Phys. Rev. Res. 2020, 2, 013313.

(97) Malola, S.; Lehtovaara, L.; Enkovaara, J.; Häkkinen, H. Birth of the localized surface plasmon resonance in monolayer protected gold nanoclusters. ACS Nano 2013, 7, 10263-10270.

(98) Zhang, R.; Bursi, L.; Cox, J. D.; Cui, Y.; Krauter, C. M.; Alabastri, A.; Manjavacas, A.; Calzolari, A.; Corni, S.; Molinari, E.; et al. How to identify plasmons from the optical response of nanostructures. ACS Nano 2017, 11, 7321-7335. 
Karlsruher Institut für Technologie

\section{Repository KITopen}

Dies ist ein Postprint/begutachtetes Manuskript.

Empfohlene Zitierung:

Müller, M. M.; Kosik, M.; Pelc, M.; Bryant, G. W.; Ayuela, A.; Rockstuhl, C.; Słowik, K. Energy-Based Plasmonicity Index to Characterize Optical Resonances in Nanostructures. 2020. The journal of physical chemistry <Washington, DC> / C, 124.

doi: $\underline{10.5445 / / R / 1000126657}$

Zitierung der Originalveröffentlichung:

Müller, M. M.; Kosik, M.; Pelc, M.; Bryant, G. W.; Ayuela, A.; Rockstuhl, C.; Słowik, K. Energy-Based Plasmonicity Index to Characterize Optical Resonances in Nanostructures. 2020. The journal of physical chemistry <Washington, DC> / C, 124 (44), 24331-24343. doi:10.1021/acs.jpcc.0c07964 